\title{
Analysis of Stiffened Penstock External Pressure Stability Based on Immune Algorithm and Neural Network
}

\author{
Wensheng Dong, ${ }^{1}$ Xuemei Liu, ${ }^{1}$ and Yunhua Li' ${ }^{1,2}$ \\ ${ }^{1}$ North China University of Water Resources and Electric Power, Zhengzhou 450011, China \\ ${ }^{2}$ School of Automation Science and Electrical Engineering, Beihang University, Beijing 100191, China \\ Correspondence should be addressed to Wensheng Dong; dongwensh@163.com
}

Received 5 November 2013; Accepted 4 January 2014; Published 19 February 2014

Academic Editor: Her-Terng Yau

Copyright (c) 2014 Wensheng Dong et al. This is an open access article distributed under the Creative Commons Attribution License, which permits unrestricted use, distribution, and reproduction in any medium, provided the original work is properly cited.

\begin{abstract}
The critical external pressure stability calculation of stiffened penstock in the hydroelectric power station is very important work for penstock design. At present, different assumptions and boundary simplification are adopted by different calculation methods which sometimes cause huge differences too. In this paper, we present an immune based artificial neural network model via the model and stability theory of elastic ring, we study effects of some factors (such as pipe diameter, pipe wall thickness, sectional size of stiffening ring, and spacing between stiffening rings) on penstock critical external pressure during huge thin-wall procedure of penstock. The results reveal that the variation of diameter and wall thickness can lead to sharp variation of penstock external pressure bearing capacity and then give the change interval of it. This paper presents an optimizing design method to optimize sectional size and spacing of stiffening rings and to determine penstock bearing capacity coordinate with the bearing capacity of stiffening rings and penstock external pressure stability coordinate with its strength safety. As a practical example, the simulation results illustrate that the method presented in this paper is available and can efficiently overcome inherent defects of BP neural network.
\end{abstract}

\section{Introduction}

Penstock is one of the important compositions in the hydroelectric power station building. It is arranged between reservoir and underground power station house [1]. In recent years, along with the construction of the large-capacity pumped storage power station and the application of highstrength materials, the structure of the penstocks is turning to huge thin-walled structure. For this structure, its stability problem under external pressure has been particularly prominent. At home and abroad, there are a lot of cases due to external pressure caused penstock buckling failure. Stability problem of hydroelectric power station penstock under external pressure has become one of the main control conditions of penstock design.

Stability analysis of stiffened penstock under external pressure includes computing of tube shell and the critical external pressure of stiffening ring. At present, the calculated method of tube shell critical load uses mainly Mises formula
[1]. Mises considered that when the instability failure tube shell between stiffen rings takes place, there will be more wave-numbers, but the amplitude is relatively small. Since there are many initial cracks between stiffened penstock and its outside concrete, the outside concrete has a smaller constraint for tube shell. The calculating of critical external load of embedded stiffened penstock can adopt the computational formula of exposed penstock and the safety coefficient can be appropriately reduced.

Actually, due to penstock exists initial defects and asymmetrical cracks, buckling penstock does not meet Mises assumption in some ways. Reference [2] proposed a calculation formula about critical load of penstock under external pressure. In the procedure of formula derivation, Lai and Fang adopted some basic assumptions, such as elastic theory, known wave numbers, and stiffener ring stiffness infinity. The formula does have a unique novelty, but due to those assumptions the application of formula is limited (when 
the ring spacing is relatively large, the calculated value is $16.9 \%$ smaller than the measured value).

In some literatures, [3-6], the derivation of penstock critical external pressure formula did not consider the influence of the external stiffened ring equivalent flange width on the critical pressure, which resulted in the computation accuracy. Liu and Ma presented a semianalytical finite element method to analyze the instability problem of the stiffened penstock [7]; it is a more objective computational method, and it can better meet the actual situation.

In this paper, a nonlinear relationship between tube shell critical external pressure and its influence factors is established by artificial immune neural network model and engages the elastic ring theory; we have studied the effects of some factors on the critical external pressure of huge thinwalled penstock (such as the pipe diameter, the pipe wall thickness, the sectional size of stiffening ring, and stiffened ring spacing) and have revealed the bearing capacity of huge thin-walled penstock plummeting reason and drastically reducing interval. By optimizing sectional size of stiffened ring and spacing among stiffened rings, we have presented an optimal design method of the bearing capacity of stiffening rings, penstock bearing capacity coordination, penstock external pressure stability, and its strength safety coordinated.

The rest of the paper is arranged as follows. In Section 2, we briefly introduce how to solve the critical load of the penstock using semianalytical element method. Section 3 discusses the simulating of the critical pressure of penstock. Section 4 studies the computation of the critical external pressure of stiffened ring. Section 5 provides one case study of one practical project. Finally, the main conclusions of the paper are inducted.

\section{Semianalytical Finite Element Method for Stability Analysis of Penstock}

In 1990, Liu and Ma proposed a semianalytic finite element computation method (SA-FEM) for the stability analysis of the penstock under external pressure [7]. This method adopts the analytical method along circumferential direction and the discrete finite element method along the axial direction, respectively. The penstock is divided into the finite cylindrical shell elements which are connected using the node circle. A typical cylindrical shell element is shown in Figure 1, where $t, R$, and $B$ denote the shell thickness, node circle radius, and element axial direction length, respectively. To facilitate study, we select the axial direction of shell middle curved surface as the $x$ coordinate (the dimensionless coordinate is $\xi$ ), the circumferential direction as the $y$ coordinate (the dimensionless coordinate $\varphi$ ), and the normal directions as the $z$ coordinate and providing positive $z$ is pointing to the direction of the curvature center. The displacement of a point in middle curved surface is $W$. The displacements of nodal $i$ and $j$ are represented by $W_{i}, \theta_{i}, W_{j}$, and $\theta_{j}$, respectively.

Defining the node displacement vector is as

$$
\{\delta\}=\left[\begin{array}{llll}
W_{i} & \theta_{i} & W_{j} & \theta_{j}
\end{array}\right]^{T}
$$

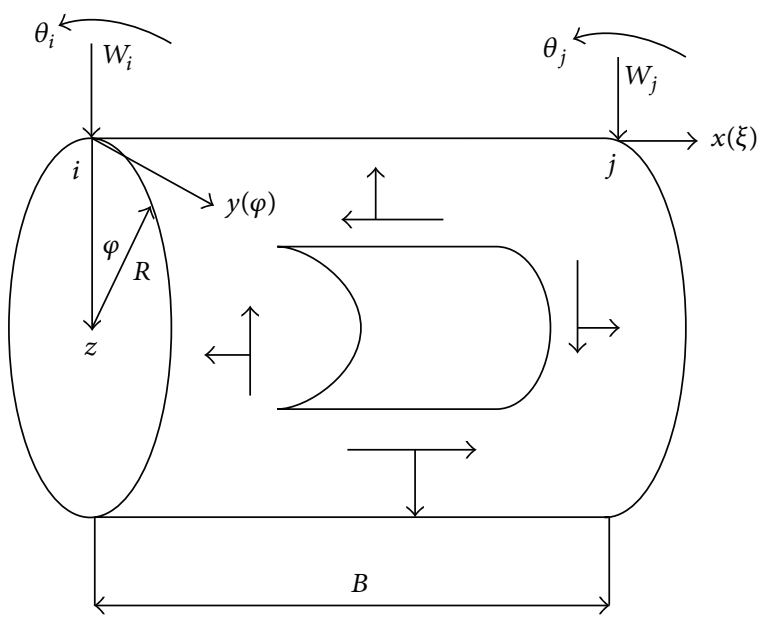

Figure 1: Cylindrical shell element.

Introducing the Hermite interpolation polynomial vector $[H]$ is as

$$
[H]=\left[H_{1}, H_{2}, H_{3}, H_{4}\right]
$$

where $H_{1}=1-3 \xi^{2}+2 \xi^{3}, H_{2}=B\left(-\xi+2 \xi^{2}-\xi^{3}\right), H_{3}=$ $3 \xi^{2}-2 \xi^{3}$, and $H_{4}=B\left(\xi^{2}-\xi^{3}\right)$; we can express the cylindrical shell element radial displacement function $X(\xi)$ along the $X$ axis as follows:

$$
X(\xi)=[H]\{\delta\} .
$$

Accordingly, the radial displacement function $W(\xi, \varphi)$ of any point on cylindrical shell element middle surface can be written as

$$
W(\xi, \phi)=X(\xi) \cos (n \phi),
$$

where $\varphi$ is central angle (radians) and $n$ is the unstable wave number along circumferential direction.

According to constraint conditions, we can see that there is neither the tensile deformation along the axial nor the shear deformation on the cylindrical shell middle surface. Therefore, from the deflection function $W$, we can derive displacement $U$ and $V$ of any point on the middle surface, so that the displacement function $\{f\}$ can be expressed as follows:

$$
\begin{aligned}
\{f\} & =\left\{\begin{array}{lll}
U & V & W
\end{array}\right\}^{T} \\
& =\left[\begin{array}{ccc}
\frac{R}{n^{2} B} \cos (n \phi) & 0 & 0 \\
0 & \frac{1}{n} \sin (n \phi) & 0 \\
0 & 0 & \cos (n \phi)
\end{array}\right]\left[\begin{array}{c}
{\left[H^{\prime}\right]} \\
{[H]} \\
{[H]}
\end{array}\right]\{\delta\},
\end{aligned}
$$

where $\left[H^{\prime}\right]$ is the first order derivative of polynomial vector $[H]$. 
According to the strain displacement relations of cylindrical shell with large deflection, we can obtain geometric equation of cylindrical shell element:

$$
\begin{aligned}
& \{\varepsilon\}=\left\{\varepsilon^{L}\right\}+\left\{\varepsilon^{N}\right\} \\
& =\left\{\begin{array}{c}
\frac{1}{B} \frac{\partial U}{\partial \xi}-\frac{z}{B^{2}} \frac{\partial^{2} W}{\partial \xi^{2}} \\
\frac{1}{R} \frac{\partial V}{\partial \phi}+\frac{2 z}{R^{2}} \frac{\partial V}{\partial \phi}-\frac{W}{R}-\frac{z}{B^{2}} \frac{\partial^{2} W}{\partial \phi^{2}} \\
\frac{1}{R} \frac{\partial U}{\partial \phi}+\frac{1}{B} \frac{\partial V}{\partial \xi}+\frac{2 z}{R B} \frac{\partial V}{\partial \xi}-\frac{2 z}{R B} \frac{\partial^{2} W}{\partial \xi \partial \phi}
\end{array}\right\} \\
& +\left\{\begin{array}{c}
\frac{1}{2 B^{2}}\left(\frac{\partial W}{\partial \xi}\right)^{2} \\
\frac{1}{2 R^{2}}\left(\frac{\partial W}{\partial \phi}\right)^{2} \\
\frac{1}{R B} \frac{\partial W}{\partial \xi} \frac{\partial W}{\partial \phi}
\end{array}\right\}
\end{aligned}
$$

where $\left\{\varepsilon^{L}\right\}$ denotes the linear strain item and $\left\{\varepsilon^{N}\right\}$ denotes the nonlinear strain item resulted by large deflection.

According to the constitutive relationships of materials and equilibrium equations of cylindrical shell element, we can set up the matrix equation of the cylindrical shell element. The stiffness matrix is composed of the elastic stiffness matrix and the equivalent geometric stiffness matrix. The elastic stiffness matrix formula of cylindrical shell element is as follows:

$$
\begin{gathered}
{\left[k_{E}\right]=\left[k_{E 1}\right]+\left[k_{E 2}\right]+\left[k_{E 3}\right]+\left[k_{E 4}\right],} \\
{\left[k_{E 1}\right]=\frac{\pi E R t\left(12 R^{2}+n^{4} t^{2}\right)}{6 n^{4} B^{3}\left(1-\mu^{2}\right)}\left[\begin{array}{cccc}
6 & -3 B & -6 & -3 B \\
-3 B & 2 B^{2} & 3 B & B^{2} \\
-6 & 3 B & 6 & 3 B \\
-3 B & B^{2} & 3 B & 2 B^{2}
\end{array}\right],} \\
{\left[k_{E 2}\right]=\frac{\pi E \mu t^{3}\left(n^{2}-1\right)}{180 R B\left(1-\mu^{2}\right)}\left[\begin{array}{cccc}
36 & -18 B & -36 & -3 B \\
-18 B & 4 B^{2} & 3 B & -B^{2} \\
-36 & 3 B & 36 & 18 B \\
-3 B & -B^{2} & 18 B & 4 B^{2}
\end{array}\right],} \\
{\left[k_{E 3}\right]=\frac{\pi E B t^{3}\left(n^{2}-1\right)^{2}}{5040 R^{3}\left(1-\mu^{2}\right)}\left[\begin{array}{cccc}
156 & -22 B & 54 & 13 B \\
-22 B & 4 B^{2} & 13 B & -3 B^{2} \\
54 & 13 B & 156 & 22 B \\
13 B & -3 B^{2} & 22 B & 4 B^{2}
\end{array}\right],} \\
{\left[k_{E 4}\right]=\frac{\pi E t^{3}\left(n^{2}-1\right)^{2}}{180 R B n^{2}(1+\mu)}\left[\begin{array}{cccc}
36 & -3 B & -36 & -3 B \\
-3 B & 4 B^{2} & 3 B & -B^{2} \\
-36 & 3 B & 36 & 3 B \\
-3 B & -B^{2} & 3 B & 4 B^{2}
\end{array}\right],}
\end{gathered}
$$

where $E$ is the modulus of elasticity $\left(\mathrm{N} / \mathrm{mm}^{2}\right), \mu$ is Poisson ratio, and the other symbols are the same as previous. The equivalent geometric stiffness matrix formula of cylindrical shell element is represented as follows:

$$
\left[k_{G}\right]=\left[k_{G x}\right]+\left[k_{G y}\right]+\left[k_{G x y}\right] \text {. }
$$

After integration operation, we found that $\left[k_{G x}\right]$ and $\left[k_{G x y}\right]$ are zero matrix, and the computing formula of $\left[k_{G y}\right]$ is as follows:

$$
\left[k_{\mathrm{G} y}\right]=-\frac{q^{e} \pi B\left(n^{2}+1\right)}{420}\left[\begin{array}{cccc}
156 & -22 B & 54 & 13 B \\
-22 B & 4 B^{2} & 13 B & -3 B^{2} \\
54 & 13 B & 156 & 22 B \\
13 B & -3 B^{2} & 22 B & 4 B^{2}
\end{array}\right]
$$

where $q^{e}$ is the radial external pressure of the element.

Adopting stiffness integration methods, penstock, we can obtain, respectively, the elastic stiffness matrix and the equivalent geometrical stiffness matrix of the overall structure. In the structural stiffness equation, introducing boundary constraint conditions, in the structural stiffness matrix, crossing out the rows and columns associated with the displacement constraints, we can get the force-balancing equation of the overall penstock as follows:

$$
\left(\left[K_{E}\right]+q\left[K_{G}^{*}\right]\right)\{\Delta\}=\{P\},
$$

where $\left[K_{E}\right]$ and $\left[K_{G}^{*}\right]$ are, respectively, elastic stiffness matrix and equivalent geometrical stiffness matrix; $\left[K_{E}\right]=\sum^{e}[K]^{e}$, $\left[K_{G}^{*}\right]=\sum^{e}\left(q^{e} / q\right)\left[K_{G}^{*}\right]^{e}$, and $e$ is element number; $q$ is external pressure of structure; $\{\Delta\}$ and $\{P\}$ are, respectively, vectors of the nodal displacement and load of the structure.

It is known from structure stability theory that characteristic equation to describe structure stability is that determinant of the overall stiffness matrix is equal to zero; that is, $\operatorname{det}\left(\left[K_{E}\right]+q\left[K_{G}^{*}\right]\right)=0$. Thus, stability problem is transformed to solve the largest eigenvalue problem of real matrix $\left(-\left[K_{E}\right]^{-1}\left[K_{G}^{*}\right]\right)$. The reciprocal of the largest eigenvalue is critical stable load.

From the above discussions, we can see that using SAFEM to solve the critical pressure of the penstock is very complicated. Actually, in practical engineering design and structure analysis, an analytical explicit formula to describe the relationship between critical pressure and structure parameters is more welcome. In order to meet this request, we provide a realization method based on neural network. Firstly, we acquire a group of the samples that adopt SAFEM to calculate critical pressure of different penstocks. Then using nonlinear mapping ability of neural network to get nonlinear relationship between critical pressure and related parameters, namely, penstock material, pipe diameter, thickness of the penstock wall, the spacing among stiffener rings, and so forth.

\section{Penstock Critical Pressure Calculating Based on Neural Network}

It is well known that neural network can approach complicated nonlinear map with very high accuracy. Immune algorithm is an evolutionary method that reflects immune system characteristic of living organisms [8,9], and it can avoid the drawbacks of traditional neural network learning algorithms. The main idea of adopting immune algorithm to design neural network is neural network structures and connection among neurons as an antibody of biological 
TABLE 1: Connection relationship table of neural nodes.

\begin{tabular}{cccccc}
\hline To & \multicolumn{5}{c}{ From } \\
& 1 & 2 & $\ldots$ & 8 & 9 \\
\hline 1 & $\times$ & $\times$ & $\ldots$ & $\times$ & $\times$ \\
2 & $\times$ & $\times$ & $\ldots$ & $\times$ & $\times$ \\
3 & $\times$ & $\times$ & $\ldots$ & $\times$ & $\times$ \\
4 & $w_{41}$ & $w_{42}$ & $\ldots$ & $\times$ & $\times$ \\
5 & $w_{51}$ & $w_{52}$ & $\ldots$ & $\times$ & $\times$ \\
6 & 0 & 0 & $\ldots$ & $\times$ & $\times$ \\
7 & $w_{71}$ & 0 & $\ldots$ & $\times$ & $\times$ \\
8 & 0 & 0 & $\ldots$ & $w_{88}$ & $\times$ \\
9 & 0 & 0 & $\ldots$ & $w_{98}$ & $w_{99}$ \\
\hline
\end{tabular}

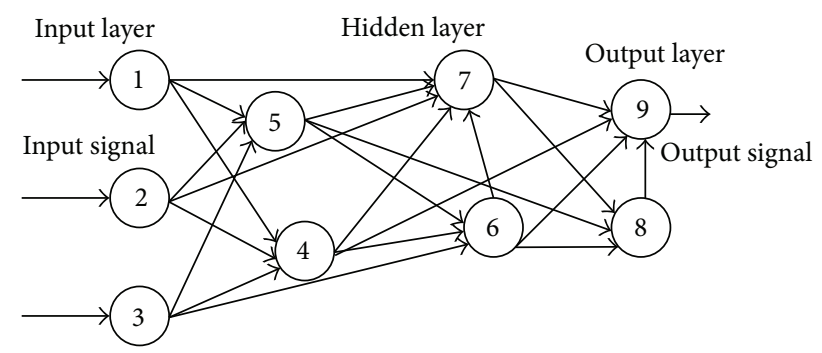

FIGURE 2: The schematic diagram of neural network.

immune system. Selection based on antibodies concentration and self-adaptive mutation operator makes antibody population continuously optimized and finally finds the best antibodies. Immune algorithm is characterized by diversity distribution of solution group and it can better overcome the shortcomings of that network structure and cryptic layer numbers defined difficultly.

3.1. Neural Network Design. In the paper, the neural network structure adopted is shown in Figure 2. The network neurons have no significant hierarchical relationship. In addition to input neurons, there are no restriction connections among neurons; each network node is assigned a serial number; the serial number of node is only used to distinguish beginning and end of directed link [10].

Deletion of Connection Edges. If the connection weights value of a connection edge is less than specified threshold range $[-0.001,0.001]$, its weights value is set to zero; that is, in the same neuron numbers circumstances, different connection form composes different network structure.

Neurons Removed. If all weights values of connection with a neuron are less than specified threshold range, deleting this neuron. The network structure and connection weights can be expressed as an equivalent matrix as Table 1. Concatenating each element of matrix constitutes an antibody. An antibody expresses a neural network structure.

\subsection{Design Steps of Neural Network Based on Immune Algorithm}

(a) Fitness Function. The antibody $\mathbf{w}^{i}$ constitutes the objective function of network:

$$
E\left(\mathbf{w}^{i}\right)=\sum_{j=1}^{n}\left|t_{j}-y_{j}\right|,
$$

where $t_{j}$ is objective output of network, $y_{j}$ is actual output, and $n$ is sample number in training sets.

Fitness function can be expressed as

$$
F\left(\mathbf{w}^{i}\right)=\frac{1}{E\left(\mathbf{w}^{i}\right)+C\left(\mathbf{w}^{i}\right)},
$$

where $C\left(\mathbf{w}^{i}\right)$ reflect impact of network complexity; it is the sum of network nodes and connections among nodes; $C\left(\mathbf{w}^{i}\right)=s\left(\mathbf{w}^{i}\right)+l\left(\mathbf{w}^{i}\right) ; l\left(\mathbf{w}^{i}\right)$ and $s\left(\mathbf{w}^{i}\right)$ represent, respectively, network connections and network nodes.

(b) Immune Selection Algorithm Based on Similarity and Vector Distance. Assuming that in a population each antibody can be represented by a one-dimensional array of $m$ elements, antibodies similarity is calculated as follows: assuming that $\mathbf{w}^{1}=\left\{w_{1}^{1}, w_{2}^{1}, \ldots, w_{m}^{1}\right\}$ and $\mathbf{w}^{2}=\left\{w_{1}^{2}, w_{2}^{2}, \ldots, w_{m}^{2}\right\}$ are any two antibodies of an antibody population with size $n$, the similarity of $\mathbf{w}^{1}$ and $\mathbf{w}^{2}$ is $d\left(\mathbf{w}^{1}, \mathbf{w}^{2}\right)$ :

$$
d\left(\mathbf{w}^{1}, \mathbf{w}^{2}\right)=\sqrt{\sum_{i=1}^{m}\left|w_{i}^{1}-w_{i}^{2}\right|},
$$

where $n$ antibodies constitute a nonempty immune set $\mathbf{W}$, the distance of two antibodies is defined as

$$
D\left(\mathbf{w}^{i}\right)=\sum_{j=1, j \neq i}^{n} d\left(\mathbf{w}^{i}, \mathbf{w}^{j}\right) .
$$

The concentration of antibody can be expressed as $\operatorname{Density}\left(\mathbf{w}_{i}\right)$ :

$$
\operatorname{Density}\left(\mathbf{w}^{i}\right)=\frac{1}{D\left(\mathbf{w}^{i}\right)} .
$$

From formula (16) we can see that the more the similarity antibodies, the greater the antibody concentration and on the contrary, the smaller the antibodies concentration.

The population Update Based on Antibody Concentration. After the parents generated offspring through mutation, according to the selection probability, random selection of individuals from the population and offspring constitutes a new population. The probability selective function is defined as follows:

$$
\begin{aligned}
P_{s}\left(\mathbf{w}^{i}\right)= & \alpha \times \operatorname{density}\left(\mathbf{w}^{i}\right)\left(1-\frac{D\left(\mathbf{w}^{i}\right)}{\sum_{i=1}^{n} D\left(\mathbf{w}^{i}\right)}\right) \\
& +\beta \frac{F\left(\mathbf{w}^{i}\right)}{\sum_{i=1}^{n} F\left(\mathbf{w}^{i}\right)},
\end{aligned}
$$


where $\alpha, \beta$ is adjustable parameter in $(0,1)$ interval, its value determined based on experience. In this paper alpha and beta value is set to 0.5 , meaning that antibody concentration and fitness have equal status during the update process of antibody population. $F\left(\mathbf{w}^{i}\right)$ is fitness of the antibody $i$.

As known from (17), the first part of right side of the equation is based on antibody concentration selection items; the higher concentration antibody has little selected chance, but the lower concentration antibody has bigger selected chance; the second part of right side of the equation is based on antibody fitness selection items; the higher fitness antibody has bigger selected chance.

(c) Generate the New Antibodies. Because the network parameters and the network structure is many to one relationship, therefore, this paper only adopts mutation operation to carry out antibodies update.

Defining mutation operator $\sigma_{i}=\sqrt{1-F\left(w^{i}\right)}$ and using it mutate all parameters of network as follows:

$$
w_{j}^{i^{\prime}}=w_{j}^{i}+\sigma_{i} \times N_{j}(0,1)
$$

where $w_{j}^{i}$ and $w_{j}^{i^{\prime}}$ are, respectively, antibodies of gene before and after mutation and $N_{j}(0,1)$ indicates random variable for each subscript $j$ re-sampling.

3.3. Simulating the Critical External Pressure of Penstock. This paper uses $[7,11,12]$ proposed calculation method to compute critical pressure and regards the computed results as the training sample of the network. The calculation model is described as follows. Penstock material is $16 \mathrm{Mn}$ (modulus of elasticity is $E=2.1 \times 10^{5} \mathrm{MPa}$, Poisson ratio $\mu=0.3$, and $\sigma_{s}=340 \mathrm{MPa}$ ). The ratio of penstock radius $r$ and shell thickness $t$ (relative tube radius $r / t$ ) is from 20 to 400; step length is 20; the ratio of rings spacing and tube radius (relative ring spacing $L / r$ ) is [ $\left[\begin{array}{lllllllll}0.1 & 0.2 & 0.3 & 0.5 & 0.8 & 1.4 & 2.0 & 3.0 & 40\end{array}\right]$. The total calculation models are 180 .

The transfers function among neurons uses s-function in the Matlab; the transfers function of output layer uses linear function. Population size is $N=50$. The simulating results are shown in Table 2.

3.4. Analysis of Calculated Results. (1) Figure 3 shows that with the increase of $r / t$ the losing stability capability of penstock under external pressure is decreased acutely. The critical external pressure decreases with increasing of $r / t$. Within $r / t=20 \sim 260$, the critical external pressure is acutely decreased; beyond the range, the change is less. For example, within $L / r=0.1 \sim 3.0, r / t$ from 20 to $260, P_{\text {cr }}$ will decrease to $0.13 \% \sim 0.16 \%$ of initial value $\left(r / t=20, P_{\text {cr }}\right)$; when the diameter of penstock is increased to a certain value, the stability power of the penstock under external pressure will change very small. For example, if $L / r=3.0$ and $r / t=400$, then the $P_{\text {cr }}$ value is only $0.02 \mathrm{MPa}$.

(2) The calculated result shows that the critical pressure $P_{\mathrm{cr}}$ of penstock decreases with relative distance of reinforcing ring $L / r$ increasing,but the influence of decreasing velocity is less than relative radius $r / t$. For example, $r / t=300$,

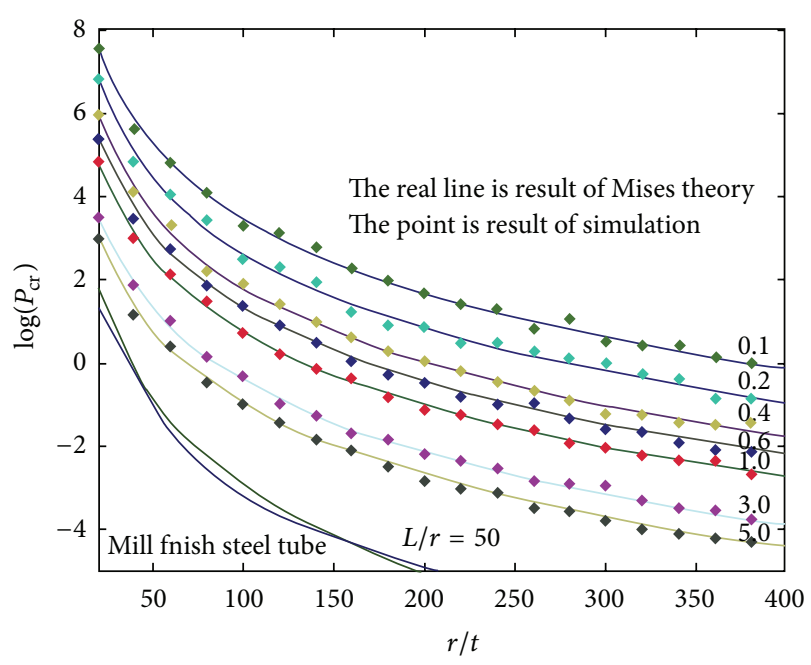

FIGURE 3: Simulating results.

TABLE 2: Simulating results of the critical external pressure $P_{\mathrm{cr}}$.

\begin{tabular}{|c|c|c|c|c|c|}
\hline \multirow{2}{*}{$\begin{array}{l}\text { Critical pressure } \\
\text { (simulation) }\end{array}$} & \multicolumn{5}{|c|}{$L / r$} \\
\hline & 0.1 & 0.2 & 0.3 & 0.5 & 0.8 \\
\hline \multicolumn{6}{|l|}{$r / t$} \\
\hline 20 & $\begin{array}{l}2024.26 \\
(2024.2)\end{array}$ & $\begin{array}{l}901.87 \\
(901.9)\end{array}$ & $\begin{array}{l}554.56 \\
(554.5)\end{array}$ & $\begin{array}{l}299.14 \\
(299.1)\end{array}$ & $\begin{array}{l}170.36 \\
(170.4)\end{array}$ \\
\hline 60 & $\begin{array}{c}119.16 \\
(119.17)\end{array}$ & $\begin{array}{l}52.05 \\
(52.14)\end{array}$ & $\begin{array}{c}31.97 \\
(31.89)\end{array}$ & $\begin{array}{c}17.43 \\
(17.56)\end{array}$ & $\begin{array}{c}10.12 \\
(10.18)\end{array}$ \\
\hline 100 & $\begin{array}{c}31.71 \\
(31.57)\end{array}$ & $\begin{array}{l}13.79 \\
(13.71)\end{array}$ & $\begin{array}{c}8.50 \\
(8.43)\end{array}$ & $\begin{array}{l}4.67 \\
(4.71)\end{array}$ & $\begin{array}{c}2.74 \\
(2.77)\end{array}$ \\
\hline 140 & $\begin{array}{l}13.23 \\
(13.38)\end{array}$ & $\begin{array}{c}5.75 \\
(5.86)\end{array}$ & $\begin{array}{c}3.55 \\
(3.47)\end{array}$ & $\begin{array}{l}1.97 \\
(2.01)\end{array}$ & $\begin{array}{l}1.16 \\
(1.14)\end{array}$ \\
\hline 180 & $\begin{array}{l}6.88 \\
(6.92)\end{array}$ & $\begin{array}{c}2.99 \\
(2.87)\end{array}$ & $\begin{array}{c}1.86 \\
(1.79)\end{array}$ & $\begin{array}{l}1.03 \\
(1.03)\end{array}$ & $\begin{array}{c}0.61 \\
(0.67)\end{array}$ \\
\hline 260 & $\begin{array}{l}2.64 \\
(2.57)\end{array}$ & $\begin{array}{c}1.16 \\
(1.23)\end{array}$ & $\begin{array}{c}0.72 \\
(0.78)\end{array}$ & $\begin{array}{l}0.40 \\
0.37)\end{array}$ & $\begin{array}{c}0.24 \\
(0.28)\end{array}$ \\
\hline 300 & $\begin{array}{l}1.82 \\
(1.81)\end{array}$ & $\begin{array}{l}0.79 \\
(0.81)\end{array}$ & $\begin{array}{l}0.49 \\
(0.51)\end{array}$ & $\begin{array}{c}0.28 \\
(0.25)\end{array}$ & $\begin{array}{c}0.16 \\
(0.16)\end{array}$ \\
\hline 360 & $\begin{array}{l}1.13 \\
(1.14)\end{array}$ & $\begin{array}{c}0.49 \\
(0.45)\end{array}$ & $\begin{array}{c}0.31 \\
(0.29)\end{array}$ & $\begin{array}{c}0.17 \\
(0.19)\end{array}$ & $\begin{array}{c}0.11 \\
(0.15)\end{array}$ \\
\hline 400 & $\begin{array}{l}0.86 \\
(0.81)\end{array}$ & $\begin{array}{c}0.37 \\
(0.36)\end{array}$ & $\begin{array}{c}0.24 \\
(0.23)\end{array}$ & $\begin{array}{c}0.13 \\
(0.11)\end{array}$ & $\begin{array}{c}0.08 \\
(0.07)\end{array}$ \\
\hline
\end{tabular}

$L / r=0.1 \sim 3.0$, and the critical external pressure $P_{\text {cr }}$ will decrease to $2.19 \% \sim 43.41 \%$ of initial value.

(3) The most effective reinforcing rings spacing and the curve of Figure 3 shows that reducing rings spacing can effectively improve the carrying capacity of critical external pressure of penstock, and while $L / r$ decrease, $P_{\mathrm{cr}}$ value and its increase ratio increase. For example, $r / t=260, L / r$ from 3.0 decrease to 0.8 and continue to decrease to 0.1 , and the increment of $P_{\text {cr }}$ is respectively $0.18 \mathrm{MPa}$ and $2.40 \mathrm{MPa}$. In other words, the reinforcing rings spacing reduces to $0.1 r$, and the average increment of $P_{\mathrm{cr}}$ value is, respectively, $0.0082 \mathrm{MPa}$ and $0.34 \mathrm{MPa}$. The latter is 41 multiples of the former. So the most effective reinforcing ring spacing should 
meet $L<0.8 r$. The reinforcing ring spacing of China's SanXia hydropower station is $L=0.32 r$.

(4) Noneffective reinforcing rings spacing Figure 3 shows that along with the stiffener ring spacing increases, the role of stiffening ring is gradually reduced, and while $L / r=40$, the $\log \left(P_{\text {cr }}\right) \sim r / t$ curves of reinforcing penstock and mill finish steel tube (the calculated formula of mill finish steel tube: $p=2 E(t / D)^{3}, D$ is diameter of tube) are almost equal. This shows that stability against external pressure of the two tubes is roughly equal; then the reinforcing ring does not possess any sustaining effect on the stiffness.

(5) The losing stability of wave numbers $n$ is a synthesis embodiment for longitudinal and circular stiffness of the penstock. With an increasing of the penstock diameter, the stiffness of circular decreases, and the losing stability of wave numbers increases. But in $X$-axial direction, with an increasing of the reinforcing ring spacing, the stiffness of $X$ axial direction of penstock decreases and also reduces the instability wave numbers $n$. For fine pitch large diameter stiffened penstock, the losing stability of wave shape shows multiwave form, but for the sparse space and small diameter shows less wave form.

(6) The design of penstock in Figure 3 shows that the curve cluster of " $\log \left(P_{\mathrm{cr}}\right) \sim n, r / t, L / r$ " is divided into two upper and lower districts by the plastic losing stability curve. If the penstock diameter and relative reinforcing rings spacing are bigger, the penstock appears elastic losing stability under smaller external pressure; $\log \left(P_{\mathrm{cr}}\right)$ value is located in the below district of the elastic losing stability curve. When the penstock diameter and relative reinforcing rings spacing are smaller, the stability of penstock under external pressure is powerful and can bear great pressure; the $\log \left(P_{\mathrm{cr}}\right)$ value is located in upon district of the elastic losing stability curve. When we design the penstock, if $r / t$ value has been determined by use, construction, and so forth, we can select appropriate $L / r$ value according to Figure 3, making the carrying capacity of penstock critical external pressure meets not only the requirements resistance to external pressure stability but also the instability curve as close as possible, in order to achieve full use of the material strength, to ensure external pressure stability and strength safety coordinated purposes.

\section{Computation of the Stiffening Ring's Critical External Pressure}

4.1. The Structure Form of Stiffening Ring. The structure forms of stiffening ring include the cross-section form of ring and the connected mode between ring and tube shell, as shown in Figure 4. As for the huge thin-wall penstock, it is advisable to adopt the structure of that both stiffening ring and penstock are rolled together as a whole which can effectively avoid penstock initial defects that caused by weld bead and uneven weld quality. The reasonable cross-section structure and dimensions of stiffening ring not only should be able to bear large external load in smaller cross-section size but also enable the critical load of tube shell close to or equal to the critical load of stiffening ring effective control range, so

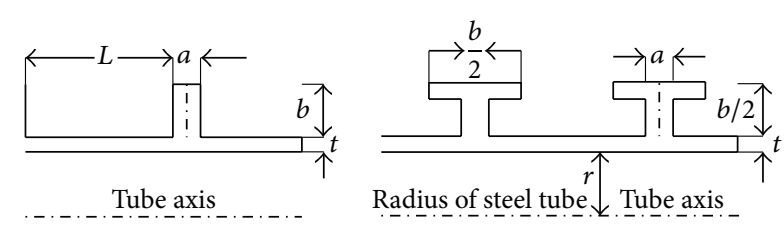

(a) Rectangle ring

(b) T-shape ring

FIGURE 4: The structure form of stiffening ring.

as to effectively improve external loads condition of structure and to facilitate construction.

On the structure that penstock and stiffening ring are rolled together as a whole, the effective control range of stiffening ring is $0.78 \sqrt{r t}$. Computing model is as follows: penstock radius $r$ is $6200 \mathrm{~mm}$, penstock shell thickness $t$ is $40 \mathrm{~mm}$, ring thickness $a$ is, respectively, 20, 40,60, and $80100 \mathrm{~mm}$, and the variation range of relative ring spacing $L / r$ is $[0.1,3.0], b / a \in[0.5,50]$. Calculating separately critical pressure of rectangle ring and T-shape ring that having the same cross-section area. The computing formula of stiffening ring critical external pressure is as follows (calculation results are shown in Table 3):

$$
P_{\mathrm{cr}}=\frac{3 E J_{k}}{R_{k}^{3} L}
$$

where $R_{k}$ is radius that is located in the gravity axis of stiffening ring effective section $(\mathrm{mm})$ and $J_{k}$ is moment of inertia that is located in the gravity axis of the stiffened ring effective section $\left(\mathrm{mm}^{4}\right)$.

For the rectangle ring

$$
\begin{gathered}
R_{k}=\frac{(a / 2)(b+t)^{2}+0.78 t^{2} \sqrt{r t}}{a(b+t)+1.56 t \sqrt{r t}}, \\
J_{k}=\frac{a}{12}(b+t)^{3}+a(b+t)\left(R_{k}-\frac{b+t}{2}\right)^{2} \\
+0.13 t^{3} \sqrt{r t}+1.56 t \sqrt{r t}\left(R_{k}-\frac{t}{2}\right)^{2} .
\end{gathered}
$$

For the T-shape ring

$$
\begin{aligned}
R_{k}= & \frac{(a b / 4)(b-a+2 t)+(a / 2)(a+t+b / 2)^{2}+0.78 t^{2} \sqrt{r t}}{(a b / 2)+a(a+t+b / 2)+1.56 t \sqrt{r t}} \\
J_{k}= & \frac{a^{3} b}{24}+\frac{a b}{2}\left(\frac{b-a}{2}-R_{k}+t\right)^{2}+\frac{a}{12}\left(\frac{b}{2}+t\right)^{3} \\
& +a\left(\frac{b}{2}+t\right) \times\left[\frac{1}{2}\left(\frac{b}{2}+t\right)-R_{k}\right]^{2}+0.13 t^{3} \sqrt{r t} \\
& +1.56 t \sqrt{r t}\left(R_{k}-\frac{t}{2}\right)^{2}
\end{aligned}
$$

The calculated results can be plotted as shown in Figure 5. 


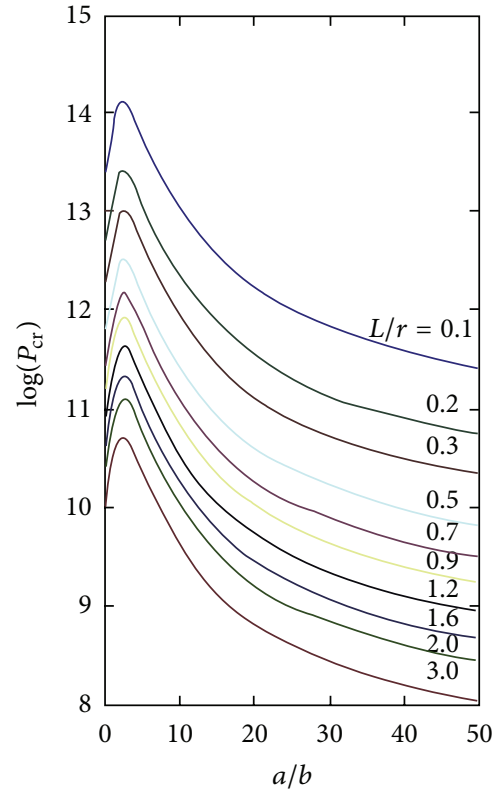

(a)

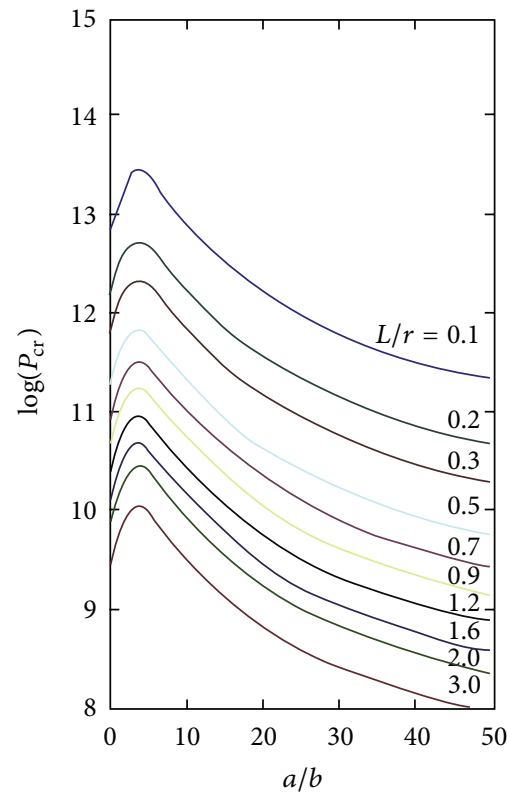

(b)

Figure 5: $\log \left(P_{\mathrm{cr}}\right) \sim b / a$ of the rectangular ring and T-shape ring.
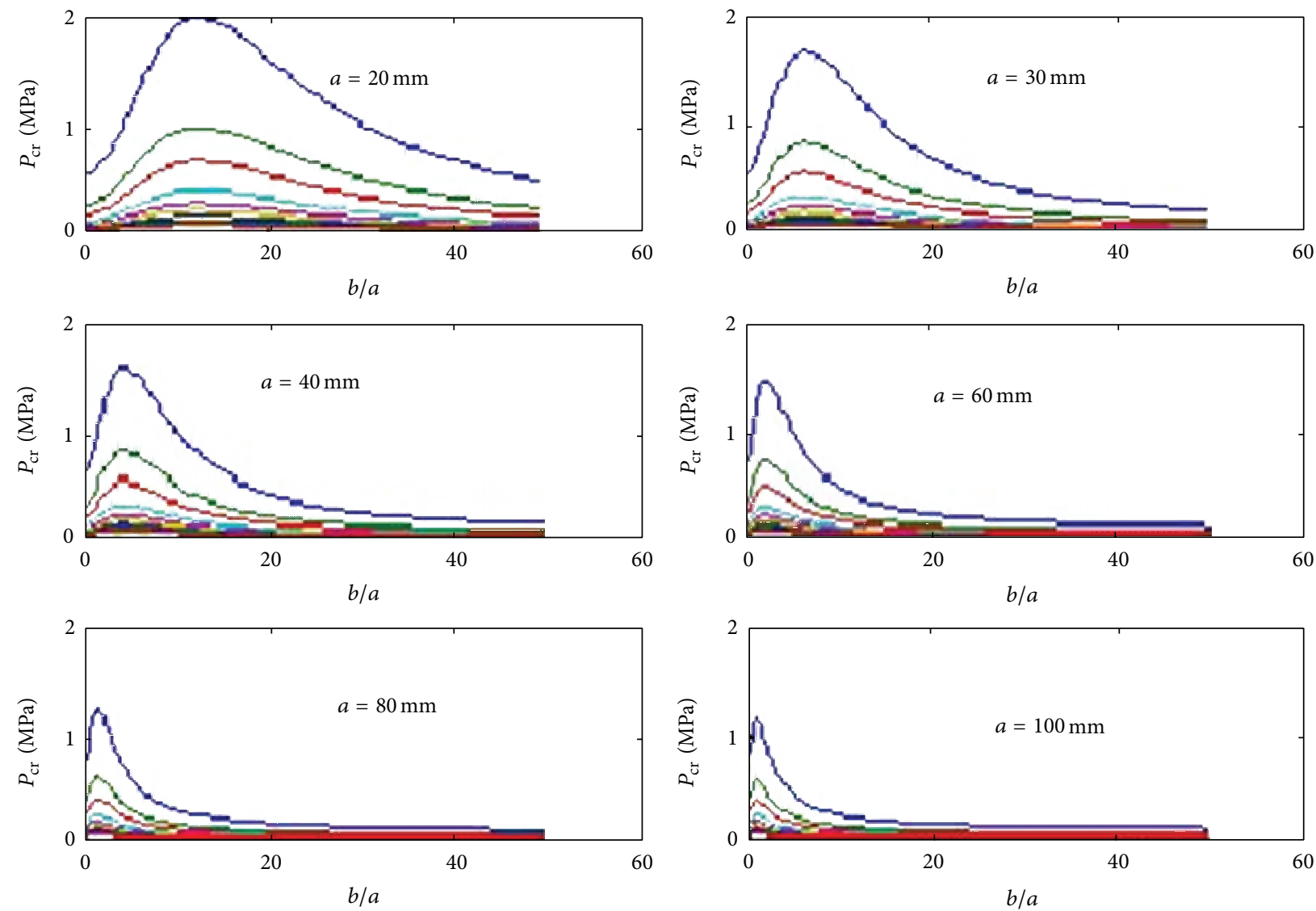

Figure 6: $P_{\mathrm{cr}}-b / a$ curve with the different $l / r$ and $a$. 
TABLE 3: The part of calculation results of rectangle ring and T-shape ring ( $a=60 \mathrm{~mm})$.

\begin{tabular}{|c|c|c|c|c|c|c|c|c|}
\hline \multirow{3}{*}{$P_{\text {cr }}(\mathrm{MPa})$} & \multicolumn{8}{|c|}{$b / a$} \\
\hline & \multicolumn{4}{|c|}{ Rectangle shaped ring } & \multicolumn{4}{|c|}{ T-shaped ring } \\
\hline & 0.5 & 1.1 & 6.5 & 16.5 & 0.5 & 1.1 & 6.5 & 16.5 \\
\hline \multicolumn{9}{|l|}{$L / r$} \\
\hline 0.1 & 657.5 & 959.1 & 746.8 & 250.9 & 388.2 & 453.0 & 574.1 & 248.4 \\
\hline 0.3 & 219.2 & 319.7 & 248.9 & 83.7 & 129.4 & 151.0 & 191.4 & 82.8 \\
\hline 2.0 & 32.8 & 47.9 & 37.3 & 12.5 & 19.4 & 22.6 & 28.7 & 12.4 \\
\hline 3.0 & 21.9 & 31.9 & 24.9 & 8.4 & 12.9 & 15.1 & 19.1 & 8.3 \\
\hline
\end{tabular}

TABle 4: Appropriate range of $b / a$ value in the conditions of different $a$.

\begin{tabular}{lcc}
\hline$a(\mathrm{~mm})$ & $\begin{array}{r}\text { With maximum } P_{\text {cr }} \\
\text { corresponding } b / a\end{array}$ & Appropriate range of $b / a$ \\
\hline 20 & 12.7 & $10-14$ \\
30 & 6.9 & $5-8$ \\
40 & 4.5 & $3.5-5.5$ \\
60 & 2.5 & $1.5-3.5$ \\
80 & 1.7 & $1.5-2.5$ \\
\hline
\end{tabular}

\subsection{Calculated Result Analysis}

(a) Stiffening Ring Reasonable Cross-Section Form. By comparing $\log \left(P_{\mathrm{cr}}\right) \sim b / a$ curve of rectangular ring and T-shape ring in Figure 5, we can see the ring with the same rings spacing and cross-section area, the rectangular ring possesses bigger $P_{\mathrm{cr}}$. The smaller the $L / r$ is, the greater this effect is. For example, for $a=60 \mathrm{~mm}, b=990 \mathrm{~mm}, L / r=0.1$, and $L / r=3.0$, the critical external pressure $P_{\mathrm{cr}}$ of rectangular ring and T-shape ring are, respectively, $(250.9,8.36) \mathrm{MPa}$ and $(248.4,8.28) \mathrm{MPa}$. While $L / r=0.1, P_{\text {cr }}$ difference between rectangular ring and $\mathrm{T}$-shape ring is $2.57 \mathrm{MPa}$, but $L / r=$ $3.0 P_{\mathrm{cr}}$ difference between rectangular ring and $\mathrm{T}$-shape ring is $0.085 \mathrm{MPa}$. It is thus clear that adopting small spacing rectangular ring is more reasonable, and the manufacture and building construction are more convenient.

(b) Stiffening Ring Appropriate Size. The computed results show the variation trend of critical external pressures of stiffening ring with $a$ and $b / a$. For the different $a$, the rising interval of $P_{\mathrm{cr}}$ with $b / a$ is different. Tables 4 and 5 show the appropriate range of $b / a$ and the critical pressure in the conditions of different $a$.

Figure 6 illustrates that under the same stiffening ring thickness, the upper limit of $P_{\mathrm{cr}}$ rising interval is unchanged, and it has no relation with the relative ring spacing. For example, when $a$ is $40 \mathrm{~mm}$ and $L / r \in[0.1,3.0]$, the corresponding $b / a$ with the maximum $P_{\mathrm{cr}}$ is 4.5 .

(c) Coupling Rule and Its Application. It can be seen from calculated results that stiffening rings with different cross section sizes, layout spacing, their bucking curves have coupling phenomenon. Therefore, by adjusting cross section sizes and layout spacing of stiffening rings, the antibuckling capacity of penstock and stiffening ring can be coordinated to the optimal state.

\section{Case Study}

5.1. Setting of Computation Conditions. The computation conditions of external pressure stability of embedded stiffened penstock in the Yachi river hydropower station include maintenance working conditions and constructing conditions. In the maintenance working conditions, normal external pressure water head $H_{1}$ is $50 \mathrm{~m}$. In the checking condition, external pressure water head $\mathrm{H}_{2}$ is $80 \mathrm{~m}$. In the constructing condition, grouting pressure of concrete $P$ is $0.3 \mathrm{MPa}$. In the above computing conditions, the maximum external pressure water head is $0.80 \mathrm{MPa}\left(\mathrm{H}_{2}=80 \mathrm{~m}\right)$, design external load is $K \times 0.80 \mathrm{MPa}, K$ is safety coefficient, and $K$ is 1.8 . In this case, the design external pressure of penstock is $1.8 \times 0.80=$ $1.44 \mathrm{MPa}$.

5.2. Stability Design of the Penstock. The penstock stability analysis and design was respectively carried out by Mises [1], Lai and Fang [2], and Liu and Ma [7]. Among them, the stiffening ring stability design method adopts formula (19) to compute. The calculate results are shown in Table 6 .

Adopting semianalytical finite element method to design penstock separately considers two situations of that simple supported role of stiffening ring and clamped role of stiffening ring.

The inside radius of penstock is $2.5 \mathrm{~m}$, stiffening ring spacing $l$ is $2.0 \mathrm{~m}$, the penstock material is $16 \mathrm{Mn}$ (elastic modulus $E$ is $210 \mathrm{GPa}$, Poisson ratio $\mu$ is 0.3 , and yield strength $\sigma$ is $325 \mathrm{MPa}$ ), and the initial crack between penstock shell and its outside concrete $\Delta$ is $0.5 \mathrm{~mm}$. Using the above several calculation methods obtain the calculation results (shown in Table 5) of external pressure stability of embedded penstock on China's YACIHE hydropower station.

The computed results show that Mises method computational results are basically situated between two computational results that calculated by semianalytical finite element method (two support forms of stiffened ring). Simulated results are close to the computational results of semianalytical finite element method with clamped stiffening ring.

Therefore, Mises calculation method can be used as main method of stiffening penstock stability design under the external pressure. Reference [2] method computed results 
TABle 5: Part of computing results of $P_{\mathrm{cr}}$ in the case of the different parameters $a$.

\begin{tabular}{|c|c|c|c|c|c|c|c|c|c|}
\hline \multicolumn{2}{|c|}{$P_{\mathrm{cr}}(\mathrm{MPa})$} & \multicolumn{8}{|c|}{$b / a$} \\
\hline$L / r$ & $a(\mathrm{~mm})$ & 0.5 & 1.5 & 1.9 & 2.1 & 2.3 & 2.9 & 3.1 & 5.5 \\
\hline \multirow{6}{*}{0.1} & 20 & 543.01 & 579.15 & 608.17 & 626.09 & 646.29 & 720.15 & 748.92 & 1195.9 \\
\hline & 30 & 558.04 & 682.73 & 772.08 & 823.19 & 877.52 & 1051.2 & 1109.9 & 1619.6 \\
\hline & 40 & 581.2 & 843.5 & 994.4 & 1069.9 & 1142.7 & 1329.6 & 1378.6 & 1480.9 \\
\hline & 60 & 657.5 & 1152.9 & 1280.3 & 1316.6 & 1336.4 & 1320.6 & 1297.9 & 886.4 \\
\hline & 80 & 766.1 & 1234.5 & 1223.0 & 1192.7 & 1153.4 & 1013.5 & 966.3 & 562.9 \\
\hline & 100 & 881.6 & 1139.7 & 1036.4 & 978.14 & 920.6 & 766.4 & 722.5 & 412.6 \\
\hline \multirow{6}{*}{0.5} & 20 & 108.60 & 115.83 & 121.63 & 125.21 & 129.26 & 144.03 & 149.78 & 239.19 \\
\hline & 30 & 111.60 & 136.64 & 54.41 & 164.64 & 175.50 & 210.25 & 221.98 & 323.92 \\
\hline & 40 & 116.2 & 168.71 & 198.9 & 213.9 & 228.5 & 265.9 & 275.7 & 296.2 \\
\hline & 60 & 131.5 & 230.6 & 256.0 & 263.3 & 267.3 & 264.1 & 259.6 & 177.28 \\
\hline & 80 & 153.2 & 246.9 & 244.6 & 238.5 & 230.7 & 202.7 & 193.26 & 112.6 \\
\hline & 100 & 176.3 & 227.9 & 207.3 & 195.6 & 184.1 & 153.3 & 144.5 & 82.5 \\
\hline \multirow{6}{*}{0.9} & 20 & 60.33 & 64.35 & 67.57 & 67.56 & 71.81 & 80.02 & 83.21 & 132.88 \\
\hline & 30 & 62.00 & 75.86 & 85.79 & 9.46 & 97.50 & 16.80 & 123.32 & 179.95 \\
\hline & 40 & 64.6 & 93.7 & 110.5 & 118.9 & 126.9 & 147.7 & 153.2 & 164.5 \\
\hline & 60 & 73.1 & 128.1 & 142.3 & 146.3 & 148.5 & 146.7 & 144.2 & 98.5 \\
\hline & 80 & 85.1 & 137.2 & 135.9 & 132.5 & 128.2 & 112.6 & 107.4 & 62.5 \\
\hline & 100 & 97.9 & 126.6 & 115.2 & 108.7 & 102.3 & 85.2 & 80.3 & 45.8 \\
\hline \multirow{6}{*}{3.0} & 20 & 18.01 & 19.31 & 20.27 & 20.87 & 21.54 & 24.00 & 24.96 & 39.86 \\
\hline & 30 & 18.60 & 22.76 & 25.74 & 27.44 & 29.25 & 35.04 & 36.99 & 53.99 \\
\hline & 40 & 19.4 & 28.1 & 33.1 & 35.7 & 38.1 & 44.3 & 45.9 & 49.4 \\
\hline & 60 & 21.9 & 38.4 & 42.7 & 43.9 & 44.5 & 44.0 & 43.3 & 29.5 \\
\hline & 80 & 25.5 & 41.2 & 40.8 & 39.8 & 38.4 & 33.8 & 32.2 & 18.8 \\
\hline & 100 & 29.4 & 37.9 & 34.5 & 32.6 & 30.6 & 25.5 & 24.1 & 13.8 \\
\hline
\end{tabular}

TABLE 6: Computational results of various calculation methods.

\begin{tabular}{|c|c|c|c|c|c|c|c|c|c|c|}
\hline \multirow{3}{*}{ Shell thickness } & \multirow{2}{*}{\multicolumn{2}{|c|}{ Mises method }} & \multicolumn{4}{|c|}{$\begin{array}{l}\text { Semianalytical finite } \\
\text { element method }\end{array}$} & \multicolumn{2}{|c|}{ Lai-Fan method } & \multicolumn{2}{|c|}{$\begin{array}{l}\text { Proposed method } \\
\text { in this paper }\end{array}$} \\
\hline & & & \multicolumn{2}{|c|}{$\begin{array}{l}\text { Stiffening ring as } \\
\text { simple supported }\end{array}$} & \multicolumn{2}{|c|}{$\begin{array}{l}\text { Stiffening ring as } \\
\text { fixed supported }\end{array}$} & \multirow[b]{2}{*}{$\begin{array}{c}P_{\mathrm{cr}} \\
\mathrm{MPa}\end{array}$} & \multirow[b]{2}{*}{$K$} & \multirow[b]{2}{*}{$\begin{array}{c}P_{\mathrm{cr}} \\
\mathrm{MPa}\end{array}$} & \multirow[b]{2}{*}{ K } \\
\hline & $\begin{array}{c}P_{\mathrm{cr}} \\
\mathrm{MPa}\end{array}$ & K & $\begin{array}{l}P_{\mathrm{cr}} \\
\mathrm{MPa}\end{array}$ & K & $\begin{array}{c}P_{\mathrm{cr}} \\
\mathrm{MPa}\end{array}$ & K & & & & \\
\hline 20 & 1.529 & 1.91 & 0.859 & 1.07 & 1.595 & 1.99 & 2.716 & 3.40 & 1.579 & 1.97 \\
\hline 22 & 1.965 & 2.46 & 1.144 & 1.43 & 2.123 & 2.65 & 3.462 & 4.33 & 2.273 & 2.84 \\
\hline 25 & 2.690 & 3.36 & 1.678 & 2.10 & 3.115 & 3.89 & 4.795 & 5.99 & 2.916 & 3.64 \\
\hline 27 & 3.266 & 4.08 & 2.114 & 2.64 & 3.924 & 4.91 & 5.846 & 7.31 & 3.784 & 4.73 \\
\hline 29 & 3.926 & 4.91 & 2.619 & 3.27 & 4.862 & 6.08 & 7.031 & 8.79 & 4.592 & 5.74 \\
\hline
\end{tabular}

TABLE 7: The calculation results of stiffening ring stability.

\begin{tabular}{|c|c|c|c|c|}
\hline \multirow{2}{*}{ Shell-thickness (mm) } & \multirow{2}{*}{ Ring-thickness (mm) } & \multirow{2}{*}{ Ring plate-high (mm) } & \multicolumn{2}{|c|}{ Stiffening ring instability } \\
\hline & & & Instability pressure (MPa) & $K$ \\
\hline 20 & 20 & 300 & 2.380 & 2.98 \\
\hline 22 & 22 & 300 & 2.685 & 3.36 \\
\hline 25 & 25 & 300 & 3.159 & 3.95 \\
\hline 27 & 27 & 300 & 3.468 & 4.36 \\
\hline 29 & 27 & 300 & 3.823 & 4.78 \\
\hline
\end{tabular}


have the great deviation. Meanwhile, this paper method is also validated.

The computed results also illustrate that when penstock shell thickness, respectively, is $20 \mathrm{~mm}$ and $22 \mathrm{~mm}$, the safe coefficient calculated by semianalytical finite element method (stiffening ring played simple-supported function) is less than 1.8 and cannot meet the requirements. However, this method considers penstock resistant external pressure capability in the case of the stiffening ring bucking, but actually penstock resistant external pressure capability should be higher than this value. The safety coefficient of other method computational results is greater than 1.8 and meet the requirements. For security purposes, penstock shell thickness, respectively, is $20 \mathrm{~mm}$ and $22 \mathrm{~mm}$ and the stiffening ring spacing can be adjusted to $1.5 \mathrm{~m}$; at this time, the calculated results by semianalytical finite element method (stiffening ring played simple-supported function), respectively, are $1.557 \mathrm{MPa}$ (safety coefficient 1.95 ) and $2.072 \mathrm{MPa}$ (safety coefficient 2.59) and meet the requirement.

5.3. The Buckling Analysis of Stiffening Ring. Set the size of stiffening ring, using formula (19), to calculate critical external pressure of the stiffened ring. The calculation results are shown in Table 7.

From Table 7 we can see that when the penstock shell radius is $2.5 \mathrm{~m}$, stiffening rings spacing is $2.0 \mathrm{~m}$, penstock thickness is $0.02 \mathrm{~m}$, and the stiffening ring height is $0.3 \mathrm{~m}$; the computational result of $P_{\text {cr }}$ is $2.380 \mathrm{MPa}, P_{\text {cr }}=2.380 \mathrm{MPa}>$ $1.44 \mathrm{MPa}(K \times 0.8)$; therefore, the stiffening ring is stable; when penstock shell thickness is $0.022 \mathrm{~m}$, the critical external pressure of stiffening ring is $2.685 \mathrm{MPa}$, and the stiffening ring stability is more reliable.

\section{Conclusions}

This paper analyzed characteristics and drawbacks of different calculation methods of penstock external pressure stability problem and proposed a simulation calculation method based on immune network. Caculation example demonstrates the feasibility of the method. The method provides a new design approach for embedded stiffening penstock external pressure stability problem in the hydropower station building engineering. The main conclusions are as follows.

(1) By analyzing the shortcomings of various calculation methods of that stiffening penstock external pressure stability problem in the current design of hydropower penstock, this paper presented simulating model of the problem. In simulation solving process, this paper adopts the immune evolutionary programming designed neural network and effectively overcomes shortcomings of hidden layer neurons and network structure which is difficult to determine in the traditional BP network, increasing convergence speed and improving global convergence capacity of the network. By comparing the results calculated by this paper calculation method and Mises calculation method (see Table 2 and Figure 3), we verify the calculation accuracy of this paper presented algorithm.
(2) The results reveal that different section sizes and different layout spacing stiffening rings, their critical pressure bucking curves have coupling phenomena. Therefore, in external pressure stability design of stiffening penstock, we can appropriately adjust stiffening rings sectional size parameters, stiffening rings spacing, and stiffening ring section structure, to make the bearing capacity of stiffening rings, penstock bearing capacity coordination, penstock external pressure stability, and its strength safety coordinated.

\section{Conflict of Interests}

The authors declare that there is no conflict of interests regarding the publication of this paper.

\section{Acknowledgments}

This research was supported by Research Programs for Innovative Research Team (in Science and Technology) of the Henan University (Grant no. 13IRTSTHN023), Innovation Scientists and Technicians Troop Construction Projects of Zhengzhou City (Grant no. 131PCXTD595), and Public Welfare industry Special Funding Research Project of Ministry of Water Resources (Grant no. 201101009).

\section{References}

[1] The Ministry of Water Resources of the People's Republic of China, "Hydroelectric power station penstock design specification," 2003.

[2] H. J. Lai and C. R. Fang, "The investigate of losing stability and breach for embedding reinforcing rings penstock under external pressure," Journal of Hydraulic Engineering, vol. 12, pp. 30-36, 1990.

[3] E. Amstutz, "Buckling of pressure-shaft and tunnel linings," International Water Power and Dam Construction, vol. 22, no. 11, pp. 391-399, 1970.

[4] S. Jacobsen, "Buckling of pressure tunnel steel linings with shear connectors," International Water Power and Dam Construction, vol. 20 , no. 6, pp. 58-62, 1968.

[5] S. Jacobsen, "Pressure distribution in steel lined rock tunnels and shafts," International Water Power and Dam Construction, vol. 29, no. 12, pp. 47-51, 1977.

[6] F. M. Svoisky and A. R. Freishist, "External pressure analysis for embedded steel penstocks," International Water Power and Dam Construction, vol. 44, no. 1, pp. 37-43, 1992.

[7] D. C. Liu and W. Y. Ma, "Research on the semi-analytical finite element methods analyzing stability of cylindrical shells external pressure," in Proceedings of the International Conference on structural Engineering and Computer, pp. 413-417, 1990.

[8] M. Srinivas and L. M. Patnaik, "Adaptive probabilities of crossover and mutation in genetic algorithms," IEEE Transactions on Systems, Man and Cybernetics, vol. 24, no. 4, pp. 656667, 1994.

[9] X.-B. Cao, K.-S. Liu, and X.-F. Wang, "Design multilayer feedforward networks based on immune evolutionary programming," Journal of Software, vol. 10, no. 11, pp. 1180-1184, 1999.

[10] M. T. Hagan and M. B. Menhaj, "Training feedforward networks with the Marquardt algorithm," IEEE Transactions on Neural Networks, vol. 5, no. 6, pp. 989-993, 1994. 
[11] W.-S. Dong, C.-H. Dang, Z.-C. Deng, and D.-C. Liu, "Stability analysis of penstock under external pressure based on GA-NN algorithms," Chinese Journal of Applied Mechanics, vol. 23, no. 2, pp. 304-307, 2006.

[12] W.-S. Dong, Z.-C. Deng, D.-C. Liu, and Y. Liang, "A relatively simple and fast method for calculating critical uniform external pressure of steel cylindrical shell structure," Journal of Northwestern Polytechnical University, vol. 24, no. 1, pp. 119-123, 2006. 


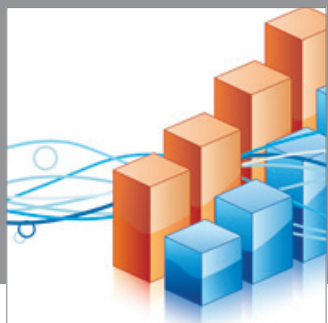

Advances in

Operations Research

mansans

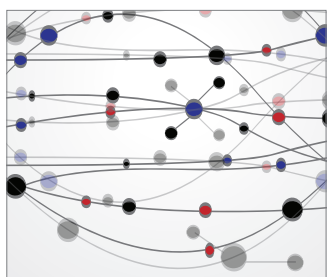

The Scientific World Journal
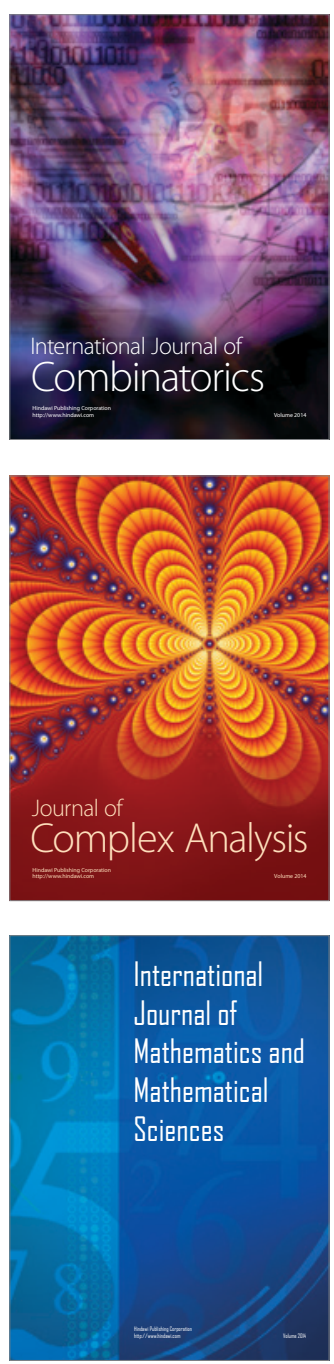
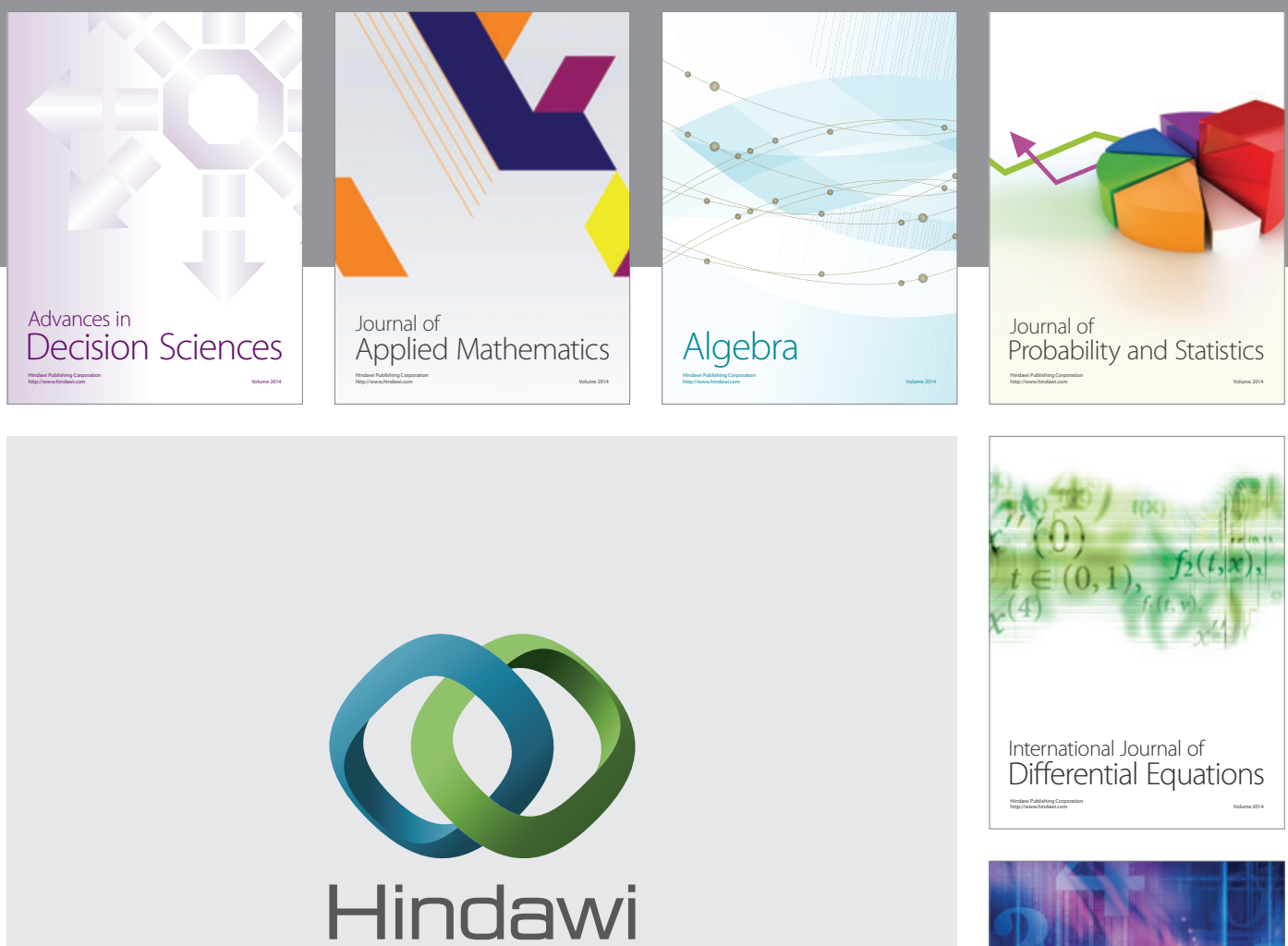

Submit your manuscripts at http://www.hindawi.com
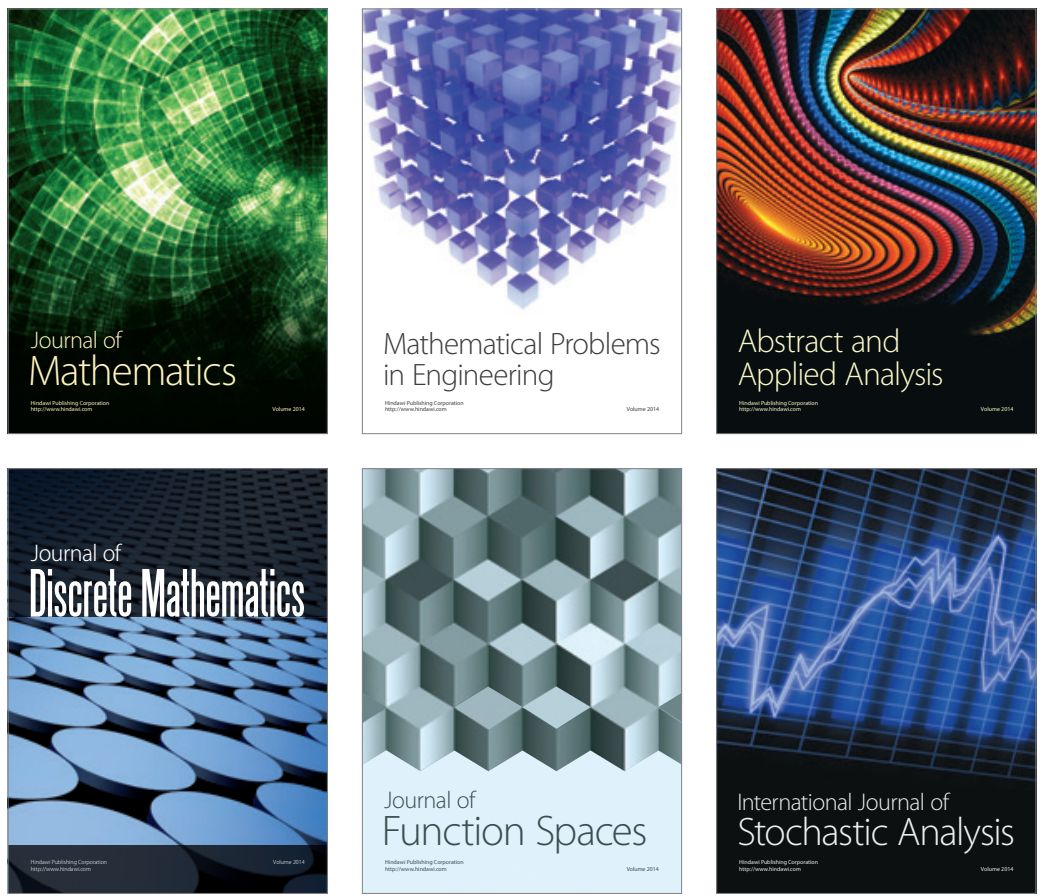

Journal of

Function Spaces

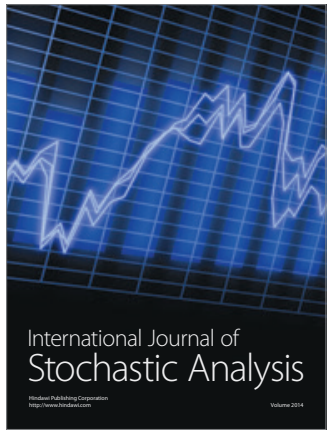

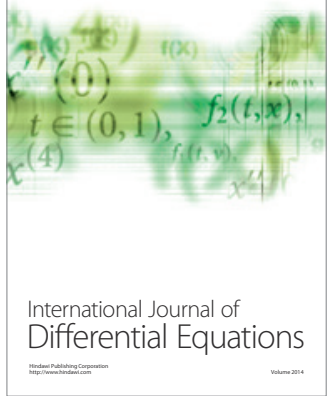
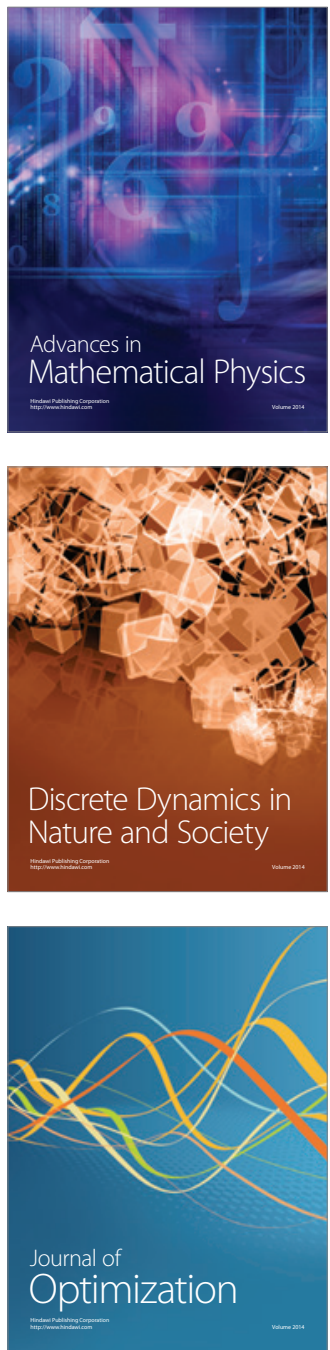